\title{
LAS TORRES DE HANOI
}

\section{HANOI TOWERS}

\section{AUTORES}

Arturo Pérez París: Universidad de Alcalá

arturo.perez@aol.com

Julio Gutiérrez: Universidad de Alcalá julio.gutierrez@mixmail.com

\section{CURRÍCULUM VITAE}

Arturo Pérez París: Alumno de la Escuela Politécnica de la Universidad de Alcalá de Henares (España). Ingeniero de Soporte Técnico en Kone Elevadores

Julio Gutiérrez: Catedrático de Física Atómica, Molecular y Nuclear en la Universidad de Alcalá de Henares. Autor de varios libros de distinta temática

\section{RESUMEN}

Nos proponemos publicar una serie de artículos encaminados a presentar, tanto a los iniciados como a los neófitos, el método numérico de resolución de ecuaciones diferenciales denominado Diferencias Finitas. Para ello, hemos creído conveniente comenzar con un sencillo ejemplo, antes de profundizar en el tema.

\section{PALABRAS CLAVE}

Torrres Hanoi - Matemáticas - Recurrencia 


\begin{abstract}
We intend to publish a series of articles designed to introduce both the initiated and the uninitiated, the numerical method for solving differential equations known as Finite Differences. To do this, we find it convenient to start with a simple example, before delving into the topic.
\end{abstract}

\title{
KEY WORDS
}

Hanoi Towers - Mathematics - Recurrence

\section{ÍNDICE}

1. Introducción

2. La recurrencia

3. Conclusión

\section{Introducción}

Nos proponemos publicar una serie de artículos encaminados a presentar, tanto a los iniciados como a los neófitos, el método numérico de resolución de ecuaciones diferenciales denominado "diferencias finitas". Para ello, hemos creído conveniente comenzar con un sencillo ejemplo, antes de profundizar en el tema.

En las ciencias de la computación y sobre todo en lo referente a su aplicación sobre sistemas de control digital, la aplicación de esta herramienta matemática es de gran 
importancia y potencia. Nos permite la síntesis de programas que realicen, a través de la adecuada ecuación, una determinada tarea que sabemos con certeza, de forma empírica, cómo sería (o habría de ser) mediante una secuencia de "n" procesos (o elementos en la jerga de los expertos).

El presente artículo pretende introducir al lector, a la manera de libro de cocina, en los misterios de la recurrencia. Está orientado, fundamentalmente, a aquellos estudiantes que, en un momento u otro de su formación, requieran de estos métodos sencillos y rápidos.

\section{La recurrencia}

Como decíamos, el estudio de la solución numérica de ecuaciones diferenciales por el método de las diferencias finitas dirigido, fundamentalmente, a una audiencia no especializada, requiere, a modo de introducción, algunas consideraciones más generales. Lo más práctico, dada su semejanza con el proceso de resolución que pretendemos exponer, es comenzar por describir un clásico rompecabezas que, si bien es muy conocido por los amantes de los juegos matemáticos, no está muy difundido. Nos estamos refiriendo a las "Torres de Hanoi", uno de los problemas que nos enfrentan con temas contrapuestos: recurrencia e iteración, unidad y diversidad. No analizaremos aquí la iteración, por no ser de relevancia en el objetivo que nos hemos propuesto. Si algún lector está interesado ello, volveríamos a insistir sobre el tema.

Aparte de su interés matemático, este rompecabezas, como otros muchos, resulta entretenido y hasta divertido, ya que confunde al neófito a la vez que le proporciona un sentimiento de satisfacción al recorrer el camino del lento progreso que le lleva a descubrir los entresijos y los misterios del pensamiento abstracto. 
Las Torres de Hanoi consisten en un tablero con tres varillas verticales. Cuando se inicia el juego, en una de las varillas (llamémosla A) se encuentra insertado un conjunto de discos de diferentes diámetros, apilados de mayor a menor, de forma que uno de mayor tamaño tiene por encima los de menor radio.

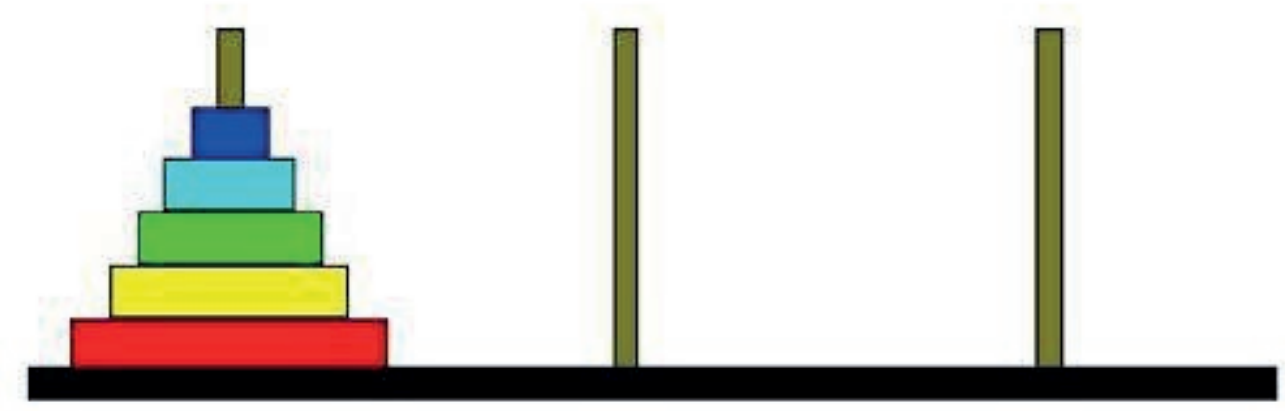

El jugador debe trasladar todos los discos a otra de las tres varillas verticales (digamos que sea a la B), con la misma estructura, es decir, la pila debe terminar con los discos gradualmente colocados de mayor a menor, con el de diámetro más pequeño en lo alto. La manipulación correspondiente ha de atenerse a las siguientes reglas:

1. Los discos han de trasladarse de uno en uno, al pasarlos de una varilla a otra.

2. Ningún disco debe tener colocado encima otro de mayor tamaño.

Obviamente, ateniéndonos a las reglas anteriores, el primer disco a mover es el más pequeño (sea a la varilla B). El siguiente movimiento no trivial consistirá en mover el disco de diámetro inmediatamente mayor a la varilla que queda libre (la C), ya que no se puede superponer al pequeño. Ahora el jugador debe decidir qué disco mover. Sólo tiene la opción de mover el disco más pequeño, pero ¿a qué varilla debe trasladarlo? Las posibilidades son devolverlo a la varilla inicial (A) o colocarlo sobre el disco inmediatamente mayor (varilla C). Aquí se enfrenta con un dilema, conocido 
como bifurcación, aunque en este caso sea simple tomar una decisión. En estadios más adelantados, podrá cometer errores que le obliguen a dar marcha atrás. Si toma la primera alternativa (devolver el disco pequeño a la varilla $\mathrm{A}$ ), no habrá conseguido nada, si opta por la segunda, tiene entonces la posibilidad de mover un tercer disco a la varilla libre.

Llamaremos "estado" a la situación del rompecabezas consistente en tener una varilla libre y las tras dos ocupadas con discos correctamente colocados. Por razones prácticas denominaremos estado 0 al inicial. Así pues, para llegar al primer estado descrito en el párrafo anterior, el jugador ha debido realizar un movimiento; y tres para llegar al segundo estado.

Si denominamos "cambio de fase" y[n] al número de movimientos a realizar para conseguir mover " $n$ " discos de una varilla a otra, correctamente colocados, tendremos:

Estado 0. Cambio de fase $y[0]=0$ movimientos.

Estado 1. Cambio de fase $y[1]=1$ movimientos.

Estado 2. Cambio de fase $y[2]=3$ movimientos.

Como llamamos cambio de fase a un número determinado de movimientos, un cambio de fase, en que $\mathrm{n}$ sea distinto de 1, puede ser considerado, a su vez, como la realización sucesiva de varios cambios de fase. Así pues, el cambio de fase y[2] equivale a tres cambios de fase $y[1]$ :

Cambio de fase $y[2]=y[1]+y[1]+y[1]$ 


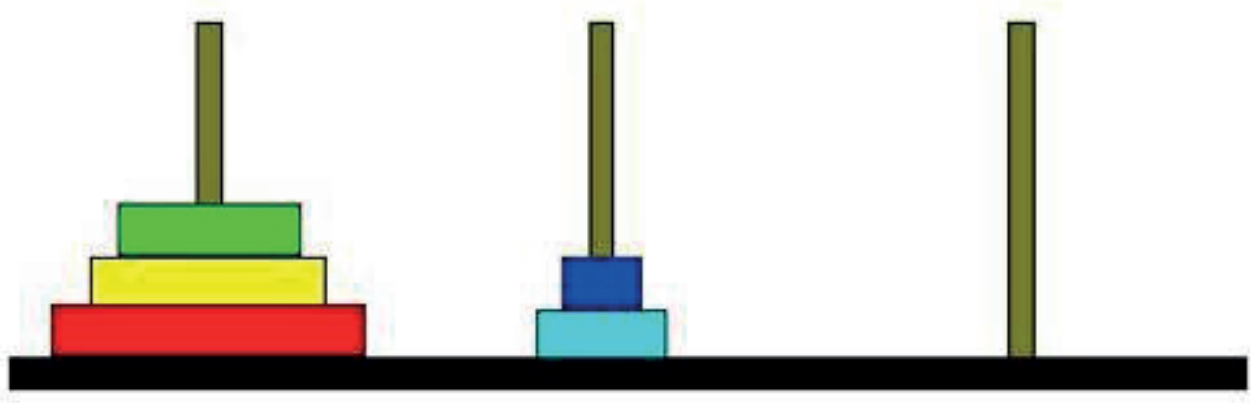

Estado 2, equivalente a un cambio de fase y[2], o tres cambios de fase y[1] El siguiente estado (3) sería el mostrado en la figura:

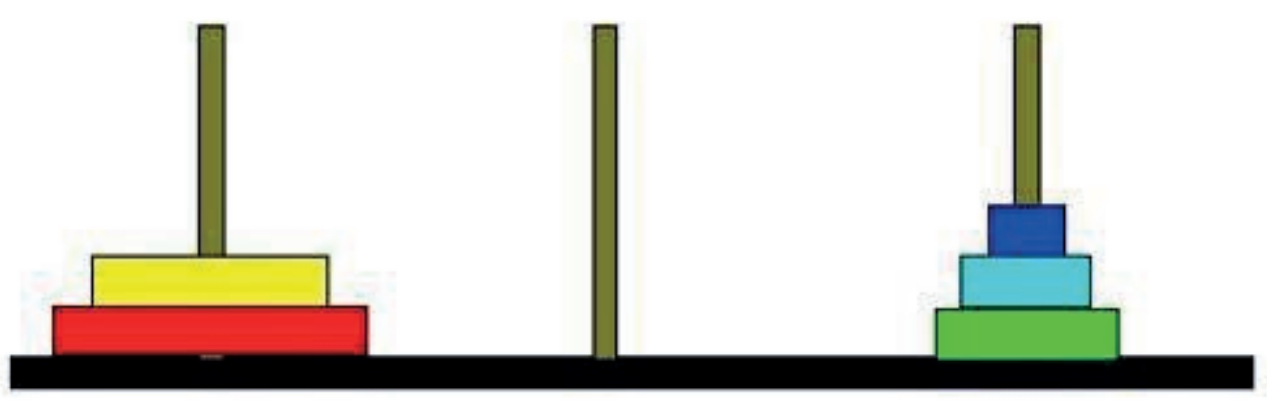

Estado 3, equivalente a un cambio de fase y[3], o siete cambios de fase y[1], o dos cambios de fase y[2] más un cambio de fase y[1]

Para ello, partiendo del segundo estado, habrá de ejecutar los siguientes movimientos: 1) Colocar el tercer disco en la varilla B; 2) Colocar el disco más pequeño en la varilla A; 3) Colocar el disco que permanece en varilla $C$, sobre el disco de la varilla $\mathrm{B}$; 4) Colocar el disco más pequeño, que ahora está en la varilla A, sobre los discos de la varilla B. En definitiva, ha realizado cuatro movimientos para pasar del segundo estado al siguiente. Es decir, para obtener, a partir de la configuración inicial, el tercer estado, debe realizar siete movimientos en total. En la notación elegida:

Cambio de fase $y[3]=7$ movimientos. 
Alternativamente, podemos describir el proceso de la forma siguiente:

a. Mover dos anillos de la varilla $\mathrm{A}$ a la $\mathrm{C}$, lo que es equivalente al cambio de fase y[2] $=3$ movimientos.

b. Mover un anillo, el tercero de la primera columna (varilla A) a la varilla B, con un único movimiento, obteniendo así una jugada equivalente a y[1] =1.

c. Por último, moveremos los dos anillos de la columna $\mathrm{C}$ hasta la varilla $\mathrm{B}$, lo equivale a realizar las jugadas correspondientes, de nuevo, a un cambio de fase y[2] = 3, completando así el transvase de anillos.

Consecuentemente, en función de los valores de las configuraciones anteriores:

$y[3]=y[2]+y[1]+y[2]=23-1=7$

Las jugadas a realizar para pasar al estado número cuatro presentan muchas posibilidades de realizar una jugada errónea, es decir, muchas bifurcaciones. Si el jugador elige siempre correctamente, no es difícil determinar, por inducción, que el número de movimientos es 24-1, es decir 15 pasos de un disco desde una columna a otra. Y en la nomenclatura de los cambios de fase:

$y[4]=y[3]+y[3]+y[1]=24-1=15$ 


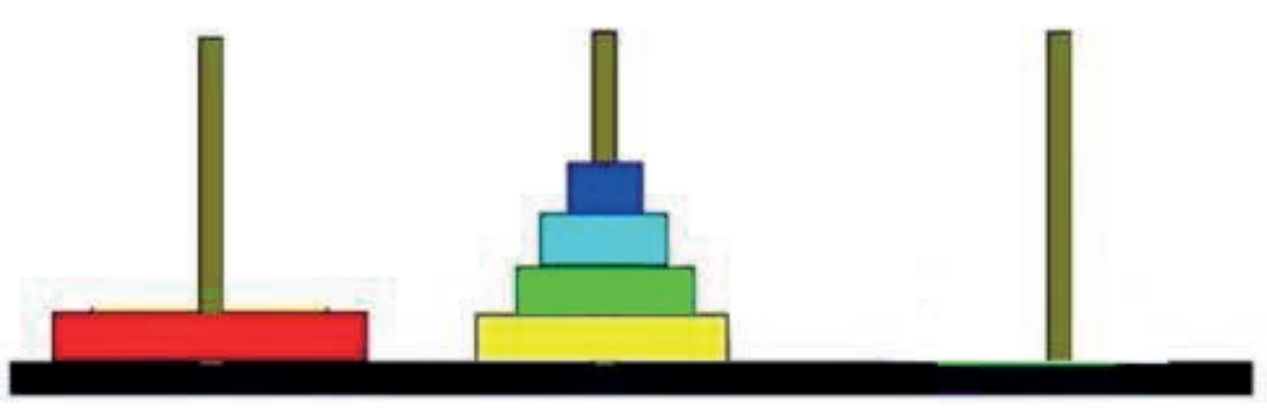

Estado 4, equivalente a un cambio de fase y[4], o dos cambios de fase y[3] más un cambio de fase $y[1]$, o quince cambios de fase $y[1]$

A raíz de estas pruebas podremos generalizar el proceso y, con ello, determinar qué número de movimientos serían precisos para pasar ' $\mathrm{n}$ ' anillos del primer poste al tercero. Sabemos que para pasar tres discos debemos pasar primero los dos que hay encima. Después nos queda el tercero que se pasará a la tercera columna de un solo movimiento. Finalmente habremos de pasar dos discos de la segunda columna a la tercera (y con esto, ya sabemos de antemano la cantidad de movimientos que nos supondrá). Por lo tanto, para pasar ' $n$ ' discos de una columna a otra, es decir para alcanzar el estado " $n$ ", determinamos por inducción: primero deberemos pasar ' $n-1$ ' discos a la segunda columna. Después el "enésimo" disco se pasará de un único movimiento a la tercera columna, quedándonos sólo por pasar los 'n-1' discos de la columna segunda a la tercera, equivalentemente:

$y[n]=y[n-1]+y[1]+y[n-1]=2 y[n-1]+y[1]$

además, como conocemos la condición inicial y[1] =1, se obtendrá finalmente:

$y[n]=2 y[n-1]+1$ 
para cualquier valor de ' $n$ ' que pertenezca al conjunto de los números enteros (nî Z). Por lo tanto, si tenemos el cambio de fase o condición inicial y[1] $=1$, la resolución de la ecuación planteada (alcanzar el estado n desde el estado 0, o lo que es igual, realizar el cambio de fase $y[n]=2 \cdot y[n-1]+1)$, pasará por la descomposición de y[n-1] en la suma de los ' $\mathrm{n}$ ' términos que conforman ese cambio de fase, p.e.:

$y[1]=1 ;$

$y[2]=2 \cdot y[1]+1=2 \cdot 1+1=3$

$y[3]=2 \cdot y[2]+1=2 \cdot 3+1=7$

$y[4]=2 \cdot y[3]+1=2 \cdot 7+1=15$

Lo que de una forma general para ' $\mathrm{n}$ ' términos se plantearía como:

$y[n]=2 n-1+2 n-2+\ldots \ldots . .+2+1=2 n-1$

Se puede apreciar que la igualdad anterior se corresponde con la suma de los ' $\mathrm{n}$ ' términos de una progresión geométrica de razón 2 ( $\mathrm{r}=2$, que consiste en el cociente entre dos términos consecutivos cualesquiera de dicha progresión), cuyo primer término es la unidad.

Nota de ayuda:

De la suma de ' $\mathrm{n}$ ' términos consecutivos de una progresión geométrica se tiene:

$\mathrm{Sn}=\mathrm{a} 1+\mathrm{a} 2+\ldots \ldots \ldots \ldots+\mathrm{an}$ 


$$
\begin{aligned}
& r \cdot S n=a 2+a 3+\ldots \ldots+a n+a n \cdot r \\
& S n-r \cdot S n=a 1-\text { an } r=> \\
& (1-r) S n=a 1-a n r=> \\
& S n=\frac{a_{1}-a_{n} \cdot r}{1-r}
\end{aligned}
$$

\section{Conclusión}

Como ya hemos mencionado, a este método de resolución de problemas se le llama "por recurrencia", ya que se busca la ley de progresión en su resolución para diferentes valores de prueba.

Hemos obtenido, usando la recurrencia, un resultado muy interesante: conociendo la solución del problema del cambio de estado 0 a un estado n-1, conocemos inmediatamente la solución para el problema del cambio de estado 0 al estado n, o lo que es lo mismo conocemos el estado $\mathrm{n}$ a través del estado n-1: en la nomenclatura de los cambios de fase

$y[n]=2 \cdot y[n-1]+1=2 n-1+2 n-2+\ldots \ldots \ldots+2+1$

Llegados aquí, para evitar la decepción del lector, al no conseguir encontrar en el comercio un tablero "Torre de Hanoi", indicamos que el rompecabezas se puede hacer perfectamente con naipes. Así, si queremos encontrar el estado descrio $n=5$, basta tomar cinco cartas consecutivas de la baraja (del as al cinco de oros, por ejemplo). Se marcan mentalmente tres puntos sobre la mesa y, sobre uno de ellos se apilan las cartas, con su cara hacia arriba, de mayor a menor, es decir, el as quedará 
en la cima. Se van trasladando naipes de un punto a otro, siguiendo las reglas del juego.

Para hacerse una idea de lo abrumadoramente largo que es este proceso de recurrencia, aunque el número de discos sea pequeño, reproducimos el relato de W.W. Rouse Ball, escrito en su libro "Mathematical Recreations and Essays":

"En el Gran Templo de la ciudad de Benarés, bajo la cúpula que señala el centro del universo, se encuentra una placa de latón con tres agujas de diamante de un codo de alto, y con un grosor igual al cuerpo de una abeja. En el momento de la Creación, Dios colocó sesenta y cuatro discos de oro en torno a una de las agujas, situado el de mayor diámetro sobre la placa de latón y de diámetro progresivamente decreciente los demás, a medida que ascendemos por la pila. Esa es la Torre de Brahma. Día y noche, sin cesar, los sacerdotes del templo vas trasladando los discos desde una aguja de diamante a otra, de acuerdo con las leyes inmutables de Brahma, que exigen que el manipulador no deba mover más de un disco por vez y que el disco debe situarse en la otra aguja de forma que los que queden debajo sean de mayor diámetro que el suyo. Cuando los sesenta y cuatro discos hayan sido transferidos completamente desde la aguja en que los colocó Dios, en el momento de la Creación, hasta una de las otras dos, la cúpula, el Templo y los Brahmanes quedarán reducidos a polvo y, con un inmenso estruendo, el universo se desvanecerá".

El universo no se ha extinguido todavía, es de suponer que el tiempo necesario para el proceso es muy largo. Dada la demostración por recurrencia que supone el cuerpo de este artículo, podemos hacer el cálculo correspondiente, para encontrar que, aún en el supuesto de que los sacerdotes hayan trabajado incansablemente a razón de un disco por segundo trasladado, harían falta 500.000 millones de años para completar la tarea. ¡Podemos dormir tranquilos! 